RIBEIRO, A. A.; SIMEÃO, M.; SOARES, J. M.; MOURA, R. S. Avaliação de modelos de estimativa da evapotranspiração de referência em Sobral, CE. Revista Agrogeoambiental, Pouso Alegre, v. 7, n. 4, p. 71-81, dez. 2015.

\title{
Avaliação de modelos de estimativa da evapotranspiração de referência em Sobral, CE
}

Aureliano Albuquerque Ribeiro ${ }^{1}$

Marcelo Simeão

Jordânia Medeiros Soares²

Regiana Santos Moura ${ }^{3}$

\section{Resumo}

Diversos métodos empíricos vêm sendo utilizados para a quantificação da evapotranspiração de referência, em virtude das dificuldades de acesso dos produtores às variáveis empregadas pelo modelo padrão de Penman-Monteith-FAO. Assim sendo, com o presente trabalho, objetivou-se avaliar, para as condições de Sobral, CE, diferentes métodos de estimativa da evapotranspiração de referência (Hargreaves-Samani, Thornthwaite, Camargo, Jensen-Haise e Ivanov), comparando-os com o método padrão de Penman-Monteith-FAO. Os dados meteorológicos foram obtidos entre o período de janeiro de 2012 a dezembro de 2012, na estação meteorológica automática do Instituto Nacional de Meteorologia (INMET), localizada em Sobral, CE. Foram coletados dados mensais de temperaturas máxima (Tmáx), média (Tméd) e mínima (Tmín) do ar, insolação, umidade relativa média do ar (URméd) e velocidade do vento a $10 \mathrm{~m}$ de altura, sendo posteriormente convertida para $2 \mathrm{~m}$. Avaliou-se a precisão dos métodos de estimativa de ETo, com base no coeficiente de correlação ( $r$ ), no índice de concordância (d) e no coeficiente de desempenho (c), o qual é o produto entre r e d. O uso do modelo de Jensen-Haise $(\mathrm{JH})$ apresentou o melhor desempenho na estimativa mensal da evapotranspiração de referência nas condições de Sobral, CE.

Palavras-chave: Agricultura irrigada. Dados meteorológicos. Jensen-Haise.

\section{Introdução}

A região Nordeste tem potencial reconhecido como grande produtora de frutas tropicais, entretanto, eventos de seca têm inibido a expressão desse potencial, o que faz da irrigação atividade obrigatória em empreendimentos agrícolas. No estado do Ceará, a fruticultura irrigada tem apresentado enorme potencial, tanto para o mercado interno quanto para o de exportação e as condições edafoclimáticas do estado favorecem a exploração da fruticultura nos perímetros irrigados (SALES, 2008). Segundo dados do IBGE (2014), em 2013 foram 545 mil hectares de frutas, com a produção de 1.650 mil toneladas, gerando um VBP (Valor Bruto da Produção) de R $\$ 1.358$ milhões, sendo o $6^{\circ}$ produtor nacional de frutas, o $1^{\circ}$ produtor de caju, o $2^{\circ}$ de coco, maracujá e melão e o $3^{\circ}$ de mamão.

Mas é a fruticultura irrigada cearense de alta tecnologia que detém os números mais expressivos da agricultura cearense, tornando o estado o $3^{\circ}$ maior exportador brasileiro de frutas frescas, exportando US $\$ 102,4$ milhões, em 2011, e US\$108,2 milhões, em 2012, depois de ter chegado a exportar US\$131 milhões, em 2008, antes da crise mundial. No primeiro semestre de 2013, já

$1 \quad$ Doutorando em Engenharia Agrícola pela Universidade Federal do Ceará.

2 Mestre em Agronomia: Solos e Nutrição de Plantas, Universidade Federal do Piauí, campus Professora Cinobelina Elvas

3 Doutoranda em Engenharia Agrícola pela Universidade Federal do Recôncavo da Bahia. 
exportou $10,5 \%$ a mais de frutas frescas do que no mesmo período do ano anterior, tendo os melões e bananas como principais produtos da pauta. Os últimos dados disponíveis sobre a agricultura irrigada cearense (2011) contabilizam 38,4 mil hectares de fruticultura irrigada, gerando $\mathrm{R} \$ 755,5$ milhões de VBP e 21,6 mil empregos diretos, sendo plantados em 6 polos de irrigação: Ibiapaba, Baixo Acaraú, Metropolitano-Curu, Baixo Jaguaribe, Centro Sul e Cariri (ADECE, 2014).

A possibilidade de estimar corretamente a evapotranspiração serve de subsídio para poder determinar a quantidade real de água a suprir o solo no monitoramento da irrigação. Desse modo, conhecer e quantificar a evapotranspiração, de forma precisa, proporcionará a possibilidade de se estimar o volume e a intensidade de água a aplicar em uma comunidade vegetal, resultando na otimização do uso dos recursos hídricos, dos equipamentos de irrigação e da energia elétrica, de modo a proporcionar a redução dos custos de produção (SALES, 2008).

A evapotranspiração de referência (ETo) pode ser determinada por métodos diretos e indiretos. O método direto (lisímetro), apesar de apresentar ótimos resultados, utiliza equipamentos de custo muito elevado, tornando inviável sua utilização no manejo da agricultura irrigada no dia a dia. Já os métodos indiretos oferecem a estimativa da ETo, sendo o método Penman-Monteith-FAO considerado padrão. Outros métodos são utilizados para se estimar a evapotranspiração de referência, como os métodos Radiação Solar, Makkink, Linacre, Jensen-Haise, Camargo, Ivanov, Hargreaves-Samani, Tanque Classe "A" e outros (BERNARDO et al., 1996).

Entretanto, antes de aplicar um método em determinado local, é preciso verificar o desempenho do solo e, quando necessário, fazer calibrações, a fim de minimizar erros de estimativa (PEREIRA et al., 2009). Por essa razão, vários trabalhos têm sido desenvolvidos no sentido de avaliar o desempenho de métodos mais simples em relação ao método padrão nas várias regiões do Brasil, tal como no Sudeste (ARAÚJO et al., 2011; SILVA et al., 2011; SOUZA et al., 2011), no Sul (BACK, 2008; SYPERRECK et al., 2008), no Nordeste (BORGES JÚNIOR et al., 2012; GONÇALVES et al., 2009) e no Centro-Oeste (OLIVEIRA et al., 2011).

Souza e Yoder (1994) compararam a Eto dos municípios de Fortaleza e Pentecoste, a partir de dados diários totalizados em mensais, no período de 1974 -1978, utilizando os modelos de Hargreaves (1974), Hargreaves (1985) e Penman-Monteith (FAO, 1991). As equações de Hargreaves (1974 e 1985) superestimam a ETo para vários meses do período, em valores que variam de uma faixa mínima de 4 a $8 \%$ e máxima entre 32 e 35\%.

Estudos realizados por Medeiros (2002) na estação meteorológica do Vale do Curu, no Centro de Pesquisa em Agroindústria Tropical pertencente à EMBRAPA de Paraipaba, CE, demonstraram que o método Priestley e Taylor (1972) e o Thornthwaite, modificado por Camargo et al. (1999), foram os que se ajustaram melhor aos valores obtidos por Penman-Monteith.

Com o presente estudo, objetivou-se avaliar, para as condições de Sobral, CE, diferentes métodos de determinação da evapotranspiração de referência, comparando-os com o método padrão de Penman-Monteith-FAO.

\section{Materiais e métodos}

O trabalho foi realizado utilizando dados meteorológicos obtidos entre o período de 1 de janeiro de 2012 a 31 de dezembro de 2012 na estação meteorológica automática do Instituto Nacional de Meteorologia (INMET), situada no município de Sobral, CE (341'03" S; 40²0'24" W; Alt. 70 m).

Conforme classificação de Köppen, o clima da região é do tipo Aw', quente, com chuvas de verão 
e máximas no outono. São dados climáticos da região: temperaturas máximas variando de $36^{\circ} \mathrm{C}$ em outubro a $31,2^{\circ} \mathrm{C}$ em maio e mínimas entre $23,2^{\circ} \mathrm{C}$ em dezembro e $21^{\circ} \mathrm{C}$ em julho; as chuvas são da ordem de $833 \mathrm{~mm}$ anuais, ocorrendo cerca de $800 \mathrm{~mm}$ de janeiro a junho, e $33 \mathrm{~mm}$ de julho a dezembro. A umidade relativa média é de 68,42mm, e a insolação anual é de 2556,0h (BRASIL, 1990).

Foram coletados dados mensais de temperaturas máxima (Tmáx), média (Tméd) e mínima (Tmín) do ar, insolação, umidade relativa média do ar (URméd) e velocidade do vento a $10 \mathrm{~m}$ de altura, sendo posteriormente convertida para $2 \mathrm{~m}$.

Os métodos utilizados para a estimativa da ETo foram: Hargreaves-Samani, Thornthwaite, Camargo, Jensen-Haise e Ivanov. Todos foram comparados ao método de Penman-Monteith, considerado padrão pela FAO.

A evapotranspiração de referência (ETo) foi calculada pelo método de Penman-Monteith-FAO (PM), equação (1):

ETo $=\frac{0,408 \Delta(R n-G)+\gamma\left(\frac{900}{(T+273)}\right) U_{2}\left(e_{s}-e_{a}\right)}{\Delta+\gamma\left(1+0,34 U_{2}\right)}$

Onde: ETo: evapotranspiração de referência $\left(\mathrm{mm}\right.$ dia $\left.^{-1}\right) ; \mathrm{Rn}$ : saldo de radiação à superfície (MJ $\left.\mathrm{m}^{-2} \mathrm{dia}^{-1}\right)$; G: fluxo de calor no solo (MJ m${ }^{-2}$ dia $\left.^{-1}\right)$; T: temperatura do ar $\left({ }^{\circ} \mathrm{C}\right) ; \mathrm{U}_{2}$ : velocidade do vento à altura de $2 \mathrm{~m}\left(\mathrm{~m} \mathrm{~s}^{-1}\right)$; $\mathrm{e}_{\mathrm{s}}$ : pressão de saturação do $\operatorname{ar}(\mathrm{kPa})$; $\mathrm{e}_{\mathrm{a}}$ : pressão de vapor atual do $\operatorname{ar}(\mathrm{kPa})$; $\left(e_{s}-e_{a}\right)$ : déficit de pressão de vapor $(\mathrm{kPa}) ; \Delta$ : declividade da curva de pressão de vapor de saturação $\left(\mathrm{kPa}{ }^{\circ} \mathrm{C}^{-1}\right)$ e $\mathrm{\gamma}$ : constante psicrométrica $\left(0,0677 \mathrm{kPa}{ }^{\circ} \mathrm{C}^{-1}\right)$.

$O$ método de Hargreaves-Samani (PEREIRA et al., 1997) foi utilizado conforme a equação (2):

$\mathrm{ETo}=0,0023 \mathrm{Ra}\left(\operatorname{Tmax}_{\mathrm{C}}-\operatorname{Tmin}_{\mathrm{C}}\right)^{0,5}(\mathrm{~T}+17,8)$

Sendo: Ra: radiação solar extraterrestre $\left(\mathrm{mm} \mathrm{dia}^{-1}\right)$; $\operatorname{Tmax}_{\mathrm{c}}$ : temperatura máxima do $\operatorname{ar}\left({ }^{\circ} \mathrm{C}\right) ; \mathrm{T}-$ $\min _{\mathrm{c}}$ : temperatura mínima do $\operatorname{ar}\left({ }^{\circ} \mathrm{C}\right)$ e T: temperatura média do $\operatorname{ar}\left({ }^{\circ} \mathrm{C}\right)$.

Realizou-se a estimativa da ETo pelo método de Thornthwaite (1948) por meio das equações 3, 4, 5, 6 e 7:

$\mathrm{ETo}=0,533 \mathrm{Fj}\left(\frac{10 \mathrm{Ta}}{\mathrm{I}}\right)^{\mathrm{a}}$

Em que: Fj = fator de correção, que varia em função do número de dias do mês (Dj ) considerado e da duração efetiva média desse dia $(\mathrm{Nj})$ obtidos, respectivamente, por:

$$
\begin{aligned}
& F=\frac{D j N j}{12} \\
& N j=2 \arccos \left(\frac{-\operatorname{tg} \Phi}{\frac{\operatorname{tg} \delta}{15}}\right)
\end{aligned}
$$

Em que: $\Phi=$ latitude, graus; $\delta=$ declinação do sol para 0 dia considerado; $\mathrm{Nj}$ = insolação máxima teórica calculada para o dia 15 de cada mês j; Ta = temperatura média mensal do ar, ${ }^{\circ} \mathrm{C}$; a = função cúbica do índice anual de calor, dada por:

$a=6,75 \times 10^{-7} I^{3}-7,71 \times 10^{-5} I^{2}+1,72 \times 10^{-2} I+0,49$

em que: I = índice de calor obtido pela soma dos 12 índices mensais (i), expresso por: 
$I=\left(\frac{\mathrm{Ta}}{5}\right)^{1,514}$

O método de Camargo (1971) foi utilizado conforme a equação (8):

$\mathrm{ETo}=0,01 \mathrm{RaTaK}$

em que: $\mathrm{Ra}=$ radiação solar extraterrestre incidente acima da atmosfera no dia 15 de cada mês $\left(\mathrm{mm} \mathrm{d}^{-1}\right)$. Para a obtenção de Ra em $\mathrm{mm} \mathrm{d}^{-1}$, dividem-se os valores em $\mathrm{MJm}^{-2} \mathrm{~d}^{-1}$ por 2,45; $\mathrm{K}=$ fator de ajuste de Camargo

O método de Jensen-Haise (1963) foi utilizado conforme a equação (9):

$\mathrm{ETo}=\mathrm{Rs}(0,025 \mathrm{Ta}+0,008)$

em que: Ta = temperatura média diária, ${ }^{\circ} \mathrm{C} ; \mathrm{Rs}=$ radiação solar global convertida em unidades de água evaporada, mm.

Realizou-se a estimativa da ETo pelo método de Ivanov (JENSEN, 1973) por meio da equação (10):

ETo $=0,006(25+\text { Tméd })^{2}\left(1-\frac{\text { URméd }}{100}\right)$

Em que Tm é a temperatura média em ${ }^{\circ} \mathrm{C}$ e UR é a umidade relativa do ar em \%.

Os valores de ETo determinados pelo modelo de Penman-Monteith foram comparados com os obtidos pelos demais modelos, utilizando análise de correlação e regressão linear para obtenção dos coeficientes da equação $(Y=a+b x)$ e do coeficiente de determinação $\left(R^{2}\right)$. Para a exatidão dos métodos empíricos, foi realizada a análise para a determinação do índice de concordância (d) de Willmott et al. (1985), do índice de desempenho (c) e da estimativa do erro padrão (EEP), descrita por Allen et al. (1986), obtidos pelas equações 11, 12 e 13, e os valores do índice "c", interpretados de acordo com a Tabela 1 (CAMARGO; SENTELHAS, 1997).

$$
d=1 \frac{\sum(P-O i)^{2}}{\sum(|P-\bar{O}|+|O i-\bar{O}|)^{2}}
$$

Onde: $\mathrm{d}$ = coeficiente de concordância; $\mathrm{Pi}=$ evapotranspiração estimada pelo método testado $(\mathrm{mm}) ; \mathrm{Oi}=$ evapotranspiração estimada pelo método padrão $(\mathrm{mm}) ; \mathrm{O}=$ média dos valores observados pelo método padrão $(\mathrm{mm})$.

$$
c=d \times r
$$

Em que: $c$ = índice de desempenho; $r=$ coeficiente de correlação

$$
E E P=\frac{\sqrt{\sum_{i=1}^{n}(P i-O i)^{2}}}{n-1}
$$

Em que: $\mathrm{EEP}=$ Estimativa do erro padrão, em $\mathrm{mm} \mathrm{dia}^{-1}$; $\mathrm{Oi}=$ valores estimados pelo método padrão, Penman-Monteith, em $\mathrm{mm} \mathrm{dia}^{-1} ; \mathrm{Pi}=$ corresponde aos valores estimados pelos métodos, em $\mathrm{mm}$ dia $^{-1} ; \mathrm{n}=$ número de observações. 
Tabela 1: Classificação do desempenho segundo o índice de confiança "c".

\begin{tabular}{lll}
\hline Valor de $c$ & Desempenho \\
\hline$\geq 0,85$ & Ótimo \\
0,76 a 0,85 & Muito bom \\
0,66 a 0,76 & Bom \\
0,61 a 0,65 & Mediano \\
0,51 a 0,60 & Sofrível \\
0,41 a 0,50 & Ruim \\
$\leq 0,40$ & Péssimo \\
\hline
\end{tabular}

Fonte: Camargo e Sentelhas (1997).

A indicação dos melhores métodos de estimativa da ETo para Sobral, CE, foram os propostos por Camargo e Sentelhas (1997).

\section{Resultados e discussão}

Dentre os métodos de estimativa da evapotranspiração de referência (ETo) avaliados, o que apresentou melhor desempenho foi o de Jensen-Haise $(\mathrm{JH})$, com desempenho Bom $(c=0,69 ; d=0,93$; EEP= $0,89 \mathrm{~mm} \mathrm{dia}^{-1}$ ) (Tabela 1 ), subestimando os valores de ETo estimados com o método de Penman-Monteith-FAO 56 (Figura 1D). Os demais métodos não apresentaram resultados satisfatórios. (Tabela 2).

Tabela 2. Coeficiente de correlação (r), estimativa do erro padrão (EEP), índice de concordância (d) e índice de confiança ou desempenho (c) na escala mensal para o período de janeiro a dezembro de 2012, em Sobral, CE.

\begin{tabular}{llllll}
\hline Métodos & EEP $\left(\mathrm{mm}_{\mathrm{dia}}{ }^{-1}\right)$ & $\mathrm{r}$ & $\mathrm{d}$ & $\mathrm{c}$ & Desempenho \\
\hline HS & 1,41 & 0,68 & 0,78 & 0,53 & Sofrível \\
TW & 1,34 & 0,56 & 0,89 & 0,49 & Ruim \\
CM & 1,39 & 0,39 & 0,90 & 0,35 & Péssimo \\
JH & 0,89 & 0,75 & 0,93 & 0,69 & Bom \\
IVN & 1,02 & 0,51 & 0,91 & 0,46 & Ruim \\
\hline
\end{tabular}

$\mathrm{HS}=$ método de Hargreaves-Samani; TW= método de Thornthwaite; $\mathrm{CM}=$ método de Camargo; JS= método de Jensen-Haise; IVN= método de Ivanov.

O melhor desempenho do método de Jensen-Haise (JH) justifica-se em virtude de ter sido desenvolvido para a aplicação nas áreas irrigadas de regiões áridas e semiáridas, semelhante às condições climáticas encontradas na área onde este estudo foi desenvolvido (MEDEIROS, 2008).

Em estudo conduzido por Gonçalves et al. (2009) para o município de Sobral, CE, o método de Jensen-Haise também apresentou índice de desempenho "c", classificado como "Bom", apresentando índice de desempenho de 0,69, superestimando os valores de ETo estimados com o método de Penman-Monteith-FAO 56. Ainda segundo os mesmos autores, o uso de métodos de estimativa da evapotranspiração que utilizam poucas variáveis para a determinação da ETo, como é o caso do referido método, que necessita apenas da temperatura média do ar, é de grande importância para a região de Sobral, pois facilita o manejo da irrigação para os produtores.

Sales (2008) recomenda que o método de Jensen-Haise (JH) deve ser indicado como opção, com certa restrição de uso na estimativa da evapotranspiração de referência, nas seguintes cidades do Ceará: Barbalha, Iguatu e Juazeiro do Norte. Pode ser utilizado sem restrições nos municípios de Acaraú, Campos Sales, Crateús, Fortaleza, Guaramiranga, Jaguaruana, Morada Nova, Paraipaba, Pentecoste, Quixeramobim, Sobral e Tauá, cidades do Ceará que apresentaram um ajuste mais significativo em relação à equação de regressão $\left(R^{2}\right.$ de 0,91 a 0,97$)$. 
O método de Hargreaves-Samani (HS) apresentou desempenho considerado Sofrível $(r=0,68$; $d=0,78$ e $c=0,53)$ e a maior estimativa de erro padrão $\left(E E P=1,41 \mathrm{~mm}\right.$.dia-1 ${ }^{-1}$, dentre os métodos avaliados, implicando a aplicação de lâminas maiores de irrigação (Tabela 2). Assim como observado para o método de Jensen-Haise (JH), o de Hargreaves-Samani (HS) também subestimou os valores de ETo estimados pelo método padrão (Figura 1A).

Também na região de Sobral, CE, Gonçalves et al. (2009) constataram que o método de Hargreaves-Samani obteve desempenho "Muito Bom". Esses resultados contrastantes justificam-se provavelmente em virtude de a escala de tempo usada ter sido distinta. No estudo realizado por Gonçalves et al. (2009), utilizaram-se dados diários, enquanto no presente estudo foram utilizados dados mensais.

Araújo et al. (2007), quando analisaram as estimativas de ETo pelos métodos de Thornthwaite, Hargreaves-Samani, Blaney-Criddle, Penman-Monteith-FAO, Tanque Classe "A" e Makkink, para o município de Boa Vista, RR, encontraram $c=0,57$, classificado também como "Sofrível" para o método de Hargreaves-Samani.
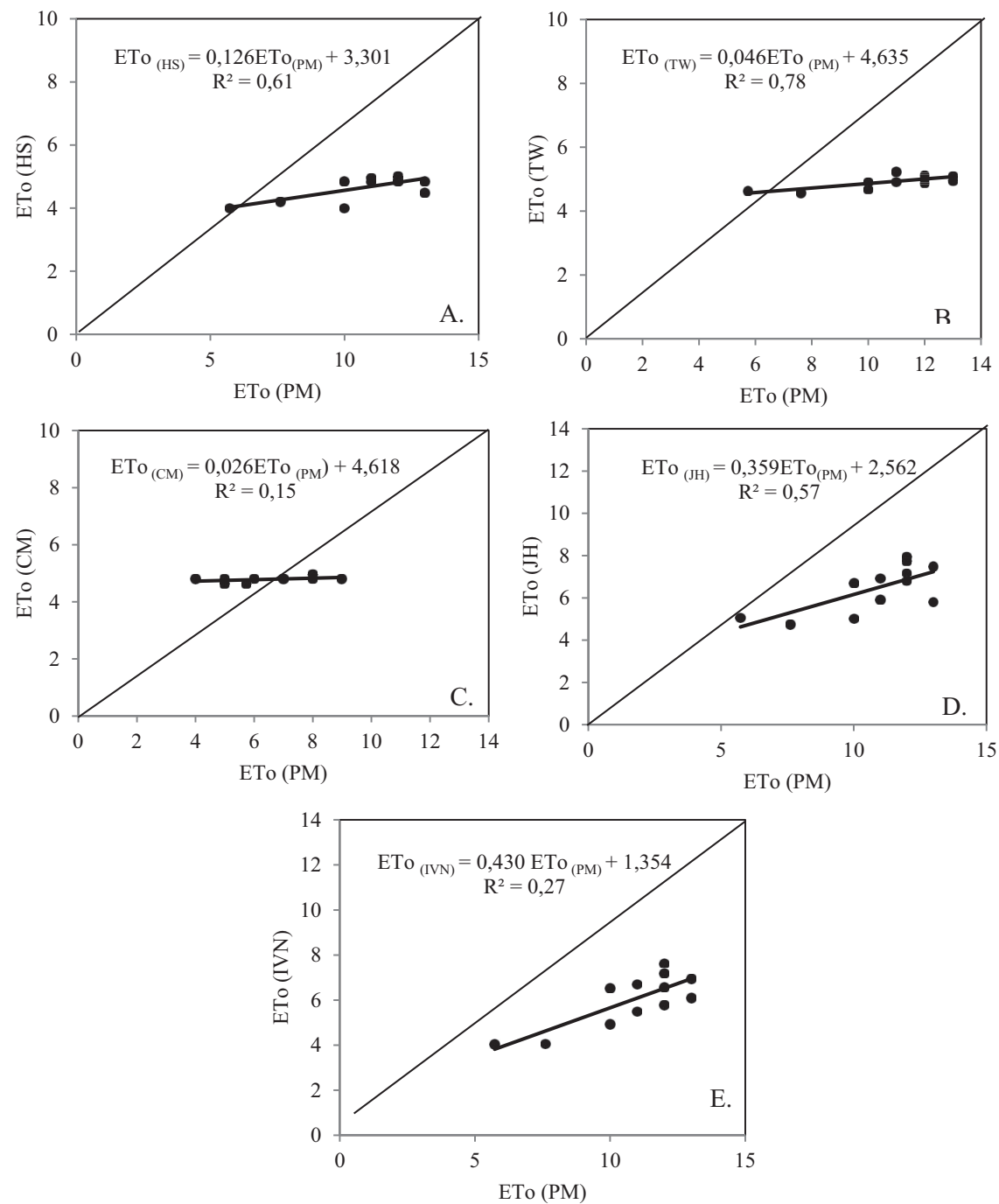

Figura 1. Regressão linear entre os valores mensais de evapotranspiração de referência (ETo) estimados pela comparação dos métodos de Hargreaves-Samani (A), Thornthwaite (B), Camargo (C), Jensen-Haise (D) e Ivanov (E), com o método padrão, Penman-Monteith, para o período de janeiro a dezembro de 2012, em Sobral, CE. Fonte: Elaboração dos autores. 
Medeiros (2002) ainda classificou o método de Hargreaves-Samani como Ruim para o município de Paraipaba, CE, subestimando os valores de ETo estimados pelo método padrão. Esse resultado é justificado pela baixa variação de temperatura e radiação solar na região litorânea do Ceará.

O método Thornthwaite (TW) foi considerado Ruim, apresentando um índice de confiança de 0,49 (Tabela 2), com estimativas que subestimaram e, em alguns momentos, superestimaram levemente a ETo estimada pelo método padrão (Figura 1B). Esse resultado era esperado, uma vez que a equação foi desenvolvida para regiões de climas úmidos (MEDEIROS, 2002), enquanto o município de Sobral, local de realização do presente estudo, apresenta clima semiárido.

Souza et al. (1995), para sete cidades do Vale do Jaguaribe e o munícipio de Crateús, CE, observaram que o método de Thornthwaite (1948) subestimou a ETo. Silva et al. (2005), em Campina Grande, PB, também observaram que o método de Thornthwaite (TW) não apresentou resultados satisfatórios na estimativa da ETo.

A equação de Thornthwaite tem sido amplamente criticada por sua natureza empírica. Porém, é bastante utilizada, já que tem como entrada apenas a temperatura (THORNTHWAITE; MATHER, 1955).

O método de Camargo (CM) apresentou valor do índice de desempenho "c", classificado como Péssimo, coeficiente de correlação " $r$ " de 0,39, índice de desempenho de 0,35, estimativa de erro padrão (EEP) de $1,39 \mathrm{~mm}$ dia $^{-1}$ (Tabela 2 ), e estimativas que subestimaram e superestimaram os valores de ETo estimados pelo método padrão em determinados momentos ao longo do ano (Figura 1C), conforme também verificado para o método de Thornthwaite (TW).

O desempenho do método de Camargo (CM) tende a ser melhor em condições de maior nebulosidade (BORGES JÚNIOR et al., 2012; SOUZA et al., 2011), condição inversa da verificada no município de Sobral, CE, justificando o baixo desempenho desse método para o referido local.

Silva et al. (2011) encontram resultados de subestimativa da ETo, utilizando o método de Camargo, no Triângulo Mineiro, mais precisamente na cidade de Uberlândia, MG. Para as condições de Rio Branco, AC, o método de Camargo apresentou desempenho classificado também como Péssimo, com valor de "c", igual a 0,33 (SOUZA, 2009).

O método de Ivanov (IVN) apresentou valor do índice de desempenho "c", classificado como Ruim, apresentando coeficiente de correlação " $r$ " de 0,51, índice de desempenho de 0,46 e estimativa de erro padrão (EEP) de 1,02 $\mathrm{mm} \mathrm{dia}^{-1}$ (Tabela 2). 0 mesmo método ainda subestimou os valores de ETo estimados pelo método padrão, conforme constatado para todos os métodos avaliados no presente estudo, com exceção dos de Thornthwaite (TW) e Camargo (CM).

Gonçalves et al. (2009) obtiveram, também para Sobral, CE, pelo mesmo método, valor do índice de desempenho "c", classificado como Sofrível. Esse comportamento, diferente do observado no presente estudo para o mesmo método, é possivelmente decorrente de a escala de tempo usada ter sido diferente nos dois estudos. Ribeiro et al. (2014) obtiveram desempenho também considerado Sofrível para o mesmo método no município de Piripiri, PI.

\section{Conclusão}

O método de Jensen-Haise ( $\mathrm{JH}$ ) apresentou o melhor desempenho na estimativa mensal da evapotranspiração de referência nas condições de Sobral, CE, sendo, portanto, recomendado o seu uso na impossibilidade de utilização do método padrão no referido local. 


\title{
Evaluation of evapotranspiration reference models in Sobral-CE
}

\begin{abstract}
Several empirical methods have been used to quantify evapotranspiration, given the difficulty of access of producers to the variables used by the standard Penman-Monteith-FAO. Thus, the present work aimed to evaluate different methods of estimating reference evapotranspiration (Hargreaves-Samani, Thornthwaite, Camargo, Jensen-Haise and Ivanov,) comparing them to the standard method Penman-Monteith-FAO under Sobral conditions. The meteorological data were obtained from January 2012 to December 2012 from the National Institute of Meteorology (INMET) automatic weather station in Sobral, CE. Monthly data on maximum (Tmax), medium (TMED) and minimum (Tmin) air temperature, sunshine, average relative humidity (URméd) and wind speed at $10 \mathrm{~m}$ height, and subsequently converted to $2 \mathrm{~m}$ were collected. We evaluated the accuracy of ETo estimation methods based on the correlation coefficient ( $r$ ), index of agreement (d) and the performance coefficient (c), which is the product between $r$ and $d$. The use of Jensen-Haise (JH) model showed the best performance in the monthly estimate of reference evapotranspiration in the conditions of Sobral, CE.
\end{abstract}

Keywords: Irrigated agriculture, Weather data, Jensen-Haise

\section{Referências}

ADECE - Agência de desenvolvimento do Estado do Ceará. Disponível em: http://www.adece.ce.gov. br/index.php/agronegocio/fruticultura.Acesso em: 30/10/2014.

ALLEN R. G.; PEREIRA, L.; RAES, D.; SMITH, M. Crop evapotranspiration: guidelines for computing crop water requirements. Rome: FAO, 1998. (FAO: Irrigation and Drainage Paper, 56).

ALLEN, R. G. A. Penman for all seasons. Journal of Irrigation and Drainage Engineering, New York, v.112, n.4, p.348-368, 1986.

ARAÚJO, W. F.; COSTA, A. A.; SANTOS, A. E. dos. Comparação entre métodos de estimativas da evapotranspiração de referência (ETo) para Boa Vista- RR. Revista Caatinga, Mossoró, v.20, n.4, p.84-88, 2007.

ARAÚJO, G. L.; REIS, E. F. dos.; MARTINS, C. A. da S.; BARBOSA, V. S.; RODRIGUES, R. R. Desempenho comparativo de métodos para a estimativa da evapotranspiração de referencia (ET ) $^{\text {) Revista }}$ Brasileira de Agricultura Irrigada, Fortaleza, v.5, n.2, p.84-95, 2011.

BACK, A. J. Desempenho de métodos empíricos baseados na temperatura do ar para a estimativa da evapotranspiração de referência em Urussanga, SC. Irriga, Botucatu, v.13, n.4, p.449-466, 2008.

BERNARDO, S.; SOUSA, E.F.; CARVALHO, J.A. Estimativa da evapotranspiração de referência (ETo), para as "áreas de baixada e de tabuleiros" da região Norte Fluminense. Campos dos Goytacazes: UENF, 1996. 14p. Boletim Técnico. 
BORGES JÚNIOR, J. C. F.; ANJOS, R. J.; SILVA, T. J. A.; LIMA, J. R. S.; ANDRADE, C. L. T. Métodos de estimativa da evapotranspiração de referência diária para a microrregião de Garanhuns, PE. Revista Brasileira de Engenharia Agrícola e Ambiental, Campina Grande, v.16, n.4, p.380-390, 2012.

BRASIL. Ministério da Agricultura. Plano Estadual dos Recursos Hídricos do Ceará: dados climatológicos de Sobral, CE (1961 - 1988). Brasília, 1990.

CAMARGO, A.P. de.; MARIN, F. R.; SENTELHAS, P. C.; PICINI, A. G. Ajuste da equação de Thornthwaite para estimar a evapotranspiração potencial em climas áridos e superúmidos, com base na amplitude térmica diária. Revista Brasileira de Agrometeorologia, Santa Maria, v.7, n.2, p.251-257, 1999.

CAMARGO, A. P.; SENTELHAS, P. C. Avaliação do desempenho de diferentes métodos de estimativa da evapotranspiração potencial no estado de São Paulo, Brasil. Revista Brasileira de Agrometeorologia, Santa Maria, v.5, n.1, p.89-97, 1997.

CAMARGO, A.P. Balanço hídrico no Estado de São Paulo. Campinas: IAC. 28p. 1971. (Boletim Técnico, 116).

FAO. Faostat Agriculture. Disponível em: <http://www.faostat.fao.org/faostat/>. Acesso em: 16 maio 1991.

GONCALVES, F. M.; FEITOSA, H. de O.; CARVALHO, C. M. de.; GOMES FILHO, R. R.; VALNIR JUNIOR, M. Comparação de métodos da estimativa da evapotranspiração de referência para o município de Sobral - CE. Revista Brasileira de Agricultura Irrigada, Fortaleza, v.3, n.2, p.71-77, 2009.

HARGREAVES, G. H.; SAMANI, Z. Reference crop evapotranspiration from temperature. Journal of Applied Engineering in Agriculture, St Joseph, v.1, n.2, p.96-99, 1985.

HARGREAVES, G. H. Estimating of potential and crop evapotranspiration. Transactions of the ASAE, St. Joseph, v.17, n.4, p.701-704, 1974.

IBGE - Instituto Brasileiro de Geografia e Estatística. Disponível em: http://www.sidra.ibge.gov.br. Acesso em: 30/10/2014.

JENSEN, M. E.; HAISE, H. R. Estimating evapotranspiration from solar radiation. J. Irrigation and Drainage. American Society Civil Engineers. v.IR4, p.15-41, 1963.

JENSEN, M.E. (Ed.). Consumptive use of water and irrigation water requirements. American Society of Civil Engineers, New York, USA, 215p.1973.

MEDEIROS, A. T. Estimativa da evapotranspiração de referência a partir da equação de Penman-Monteith, de medidas lisimétricas e de equações empíricas em Paraipaba, CE. 2002. 103p. Tese (Doutorado em Agronomia) - Escola Superior Luiz de Queiroz, Piracicaba.

MEDEIROS, P. V. Análise da evapotranspiração de referência a partir de medidas lisimétricas e ajuste estatístico de estimativas de nove equações empiríco-teóricas com base na equação de Penman-Monteith. 2008. 241p. Dissertação (Mestrado em Hidráulica e Saneamento) - Universidade de São Paulo, São Carlos. 
OLIVEIRA, G. Q. de.; LOPES, A. da S.; JUNG, L. H.; NAGEL, P. L.; BERTOLI, D. M. Desempenho de métodos de estimativa da evapotranspiração de referencia baseadas na temperatura do ar, em Aquidauana - MS. Revista Brasileira de Agricultura Irrigada, Fortaleza, v.5, n.3, p.224-234, 2011.

PEREIRA, D. R.; Desempenho de métodos de estimativa da evapotranspiração de referência para a região da Serra da Mantiqueira, MG, Ciência Rural, Santa Maria, v.39, n.9, p.2488-2493, dez. 2009.

PEREIRA, A. R.; VILLA NOVA, N. A.; SEDIYAMA, G. C. Evapotranspiração. Piracicaba: FEALQ, 1997. 183p.

PRIESTLEY, C. H. B.; TAYLOR, R. J. On the assessment of surface heat flux and evaporation using large-scale parameters. Monthly Weather Review, Madson, v.100, p.81-92, 1972.

RIBEIRO, A. A.; ANDRADE JÚNIOR, A. S.; SILVA, E. M.; SIMEÃO, M.; MOURA, R. S. Métodos empíricos de estimativa da evapotranspiração de referência para Piripiri, Piauí. In: CONGRESSO BRASILEIRO DE ENGENHARIA AGRÍCOLA, Campo Grande, 2014. Anais... Campo Grande: Sociedade Brasileira de Engenharia Agrícola. 2014.

SALES, J. C. Caracterização climática e comparação de métodos de estimativa de evapotranspiração de referência para regiões do Estado do Ceará. 2008. 212p. Tese (Doutorado em Irrigação e Drenagem). Universidade Estadual Paulista Júlio de Mesquita Filho, Botucatu.

VICENTE DE P. R. DA SILVA; ADELGICIO F. BELO FILHO; BERNARDO B. DA SILVA; JOÃO H. B. DA C. CAMPOS. Desenvolvimento de um sistema de estimativa da evapotranspiração de referência. Revista Brasileira de Engenharia Agrícola e Ambiental, Campina Grande, v. 9, n. 4, p. 547-553, 2005.

SILVA, V. J. da.; CARVALHO, H. de P.; SILVA, C. R. da.; CAMARGO, R. de.; TEODORO, R. E. F. Desempenho de diferentes métodos de estimativa da evapotranspiração de referência diária em Uberlândia, MG. Bioscience Journal, Uberlândia, v.27, n.1, p.95-101, 2011.

SYPERRECK, V. L. G.; KLOSOWSKI, E. S.; GRECO, M.; FURLANETTO, C. Avaliação de desempenho de métodos para estimativas de evapotranspiração de referência para a região de Palotina, Estado do Paraná. Acta Scientiarum: Agronomy, v.30, p.603-609, 2008.

SOUZA, M. L. A. de. Comparação de métodos de estimativa da evapotranspiração de referência (ETo) em Rio Branco, Acre. 2009. 75p. Dissertação (Mestrado)- Universidade Federal do Acre, Rio Branco.

SOUZA, A.P. de.; CARVALHO, D. F. de.; SILVA, L. B. D. da.; ALMEIDA, F. T. de.; ROCHA, H. S. Estimativas da evapotranspiração de referência em diferentes condições de nebulosidade. Pesquisa Agropecuária Brasileira, Brasília, v.46, n.3, p.219-228, 2011.

SOUZA, F. de.; YODER, R. Estimation in the northeast of Brazil: Hargreaves or man-Monteith equation. St. Joseph: ASAE, 1995. 6 p.

SOUZA, F. J. Comparação de diferentes modelos utilizados para estimar a evapotranspiração de referência (ETo) nas condições do Brejo Paraibano. In: CONGRESSO BRASILEIRO DE ENGENHARIA AGRíCOLA. Viçosa, 1995. Anais... Viçosa: Sociedade Brasileira de Engenharia Agrícola. 1995. 
THORNTHWAITE, C. W.; MATHER, J. R. The water balance: publications in climatology. New Jersey: Drexel Institute of Technology, 1955.

THORNTHWAITE, C. W.; MATHER, J. R. An approach toward a rational classification of climate. The Geographycal Review, New York, v.38 n.1, p.55-94, 1948.

WILLMOTT, C. J.; CKLESON, S.G.; DAVIS, R. E. Statistics for the evaluation and comparision of models. Journal of Geophysical Research. Ottawa, v.90, n.C5, p. 8995 - 9005, 1985.

\section{Histórico editorial}

Submetido em: 02/12/2013

Aceito em: 16/12/2014 